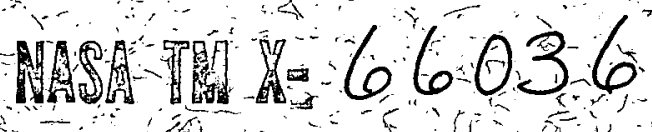

\section{THEORETICAL AND OBSERVATIONAL ANALYSIS OF SPACECRAFT FIELDS}

\section{F. M. NEUBAUER \\ K. H. SCHATTEN}

\section{SEPTEMBER 1972}

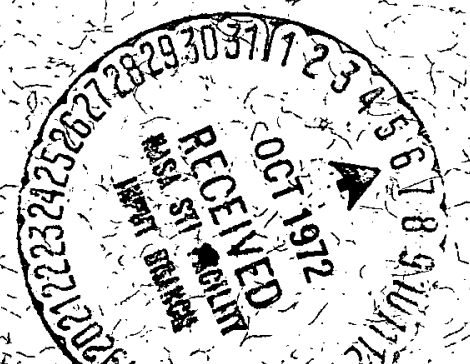

\section{GODDARD SPACE FLIGHT CENTER}

\section{GREENBELT MARYLAND}

(NASA-TM-X-66036) THEORETICAL AND

N $72-32654$

OBSERVATIONAL ANALYSIS OF SPACECRAFT FIELDS

F.M. Neubauer, et al (NASA) Sep. 1972

$23 \mathrm{p}$

CSCL $20 \mathrm{C}$ 


\title{
THEORETICAL AND OBSERVATIONAL ANALYSIS OF SPACECRAFT FIELDS
}

\author{
by \\ F. M. Neubauer ${ }^{1}$ \\ K. H. Schatten 2 \\ Laboratory for Extraterrestria1 Physics \\ NASA/Goddard Space Flight Center \\ Greenbelt, Maryland 20771
}

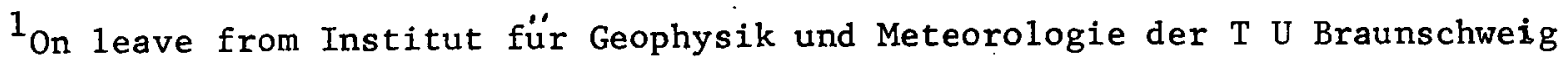
Germany

${ }^{2}$ Now at Physics Dedortment, Victoria University of Wellington, Wellington, New Zeal and. 
Abstract

In order to investigate the nondipolar contributions of spacecraft magnetic fields a simple magnetic field model is proposed. This mode1 consists of a number $\mathrm{N}$ of randomly oriented dipoles of strength $\mathrm{M}_{k}$ in a given volume. Two sets of formulas are presented which give the rms-multipole. field components, firstly for isotropic orientations of the dipoles at given positions and secondly for isotropic orientations of the dipoles distributed uniformly throughout a cube or sphere. The statistical results for a cube $\left(\right.$ size $\left.=8 \mathrm{~m}^{3}\right)$ together with individual examples computed numerically show the following features: Beyond about 2-3m distance from the center of the cube, the field is dominated by an equivalent dipole. The magnitude of the magnetic moment of the dipolar part is approximated by $\mathrm{N}^{1 / 2} \mathrm{M}$ for equal magnetic moments or generally by the Pythagorean sum of the dipole moments. The radial component is generaliy greater than either of the transverse components for the dipole portion as well as for the nondipolar field contributions. Part of the results of the simple model is compared with magnetic field mappings of IMP-H and to a lesser extent of Mariner 5 and found generally consistent with the measurements. 


\section{INTRODUCTION}

A variety of magnetometers has been employed for measurements of magnetic fields in space. Ness (1970) reviews these instruments, their uses and many of their associated problems. One problem that has been dea1t with mostly from an experimental viewpoint is that of spacecraft associated fields.

Observations of the magnetic field in space have been limited, in part, by the spacecraft associated magnetic fields. These fields originate from spacecraft power sources (solar arrays, batteries), spacecraft structure, electrical distribution system, subsystem electronics, and scientific experiments. General1y, to minimize the effect of these magnetic fields, the magnetometer sensors have been positioned as remotely as possible from the main body of the spacecraft, subject to cost and engineering constraints. In addition, magnetic cleanliness programs have been carried through with varying success to reduce the spacecraft field by selecting nonmagnetic electronic parts, "backwiring" of solar arrays and other self-compensating wiring and component techniques.

Recently a method to continuously estimate the spacecraft magnetic field in flight. has been suggested by Ness et a1. (1971), which may further reduce spacecraft field associated errors. Using two magnetometers this method may allow the complete removal of discrete spacecraft field events and removal of the dipole component of the slowly changing spacecraft field. Errors due to the slowly varying higher order moments of the spacecraft field may still contribute to the spacecraft field associated part of the overall error.

Thus it becomes important to gain an understanding of the significance of the non-dipolar terms of the spacecraft magnetic field. The lowest order 
multipole fields are usually the most significant ones in affecting the magnetic field observations, since the magnetometers are generally located moderately far from the spacecraft body. We shall attempt to obtain a simple magnetic model for the spacecraft and compare the results with observations obtained during the tests of real spacecraft. More specifically, in the model calculations we consider the various spacecraft field contributions both for individual models and from a statistical point of view. 
II. THEORETICAL STATISTICAL ANALYSIS OF SPACECRAFT FIELDS

In this section we present some analytical statistical calculations to gain an understanding of the relative magnitude of the lower multipole contributions to a spacecraft field. To do this we first have to choose a model for the spacecraft field.

In our simple model we approximate the spacecraft field by $\mathrm{N}$ dipoles of magnetic moments $\underline{M}_{k}$ with $\left.\right|_{-k} \mid=M_{k}$ and positions $\underline{x}_{k}$ inside the volume $V_{\text {sc. }}$ Each dipole may represent a spacecraft subsystem or part of a subsystem. We also note, that the non-dipolar field of a given source can be represented by a suitable set of dipoles to achieve any wanted accuracy.

If only the magnitude $M_{k}$ of the dipole moments and their positions $\underline{x}_{k} k-1, \ldots N$ were given, then to describe any net field there would be an infinite number of individual models, which are distinguished by the different orientations of their N dipoles. Assuming an isotropic distribution of the dipole orientations with no correlations between these orientations we obtain the following rms values for the spherical components $B_{R}$ parallel to the magnetometer boom and $\sqrt{B_{\Phi}^{?}+B_{\theta}^{2}}$ perpendicular to the boom as a furction of distance $r$ along the boom:

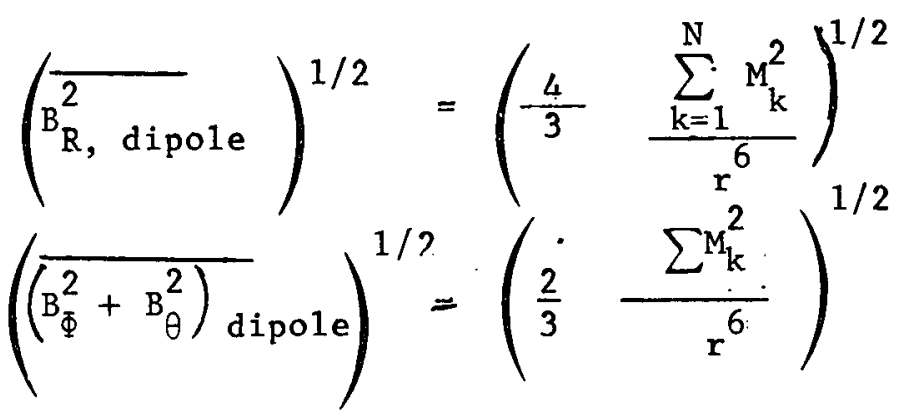




$$
\begin{aligned}
& \left(\overline{B_{R}^{2}, \text { quadrupole }}\right)^{1 / 2}=\frac{1}{r^{4}}\left(\sum M_{k}^{2}\left(9 x_{k}^{2}+3 r_{k}^{2}\right)^{1 / 2}\right.
\end{aligned}
$$

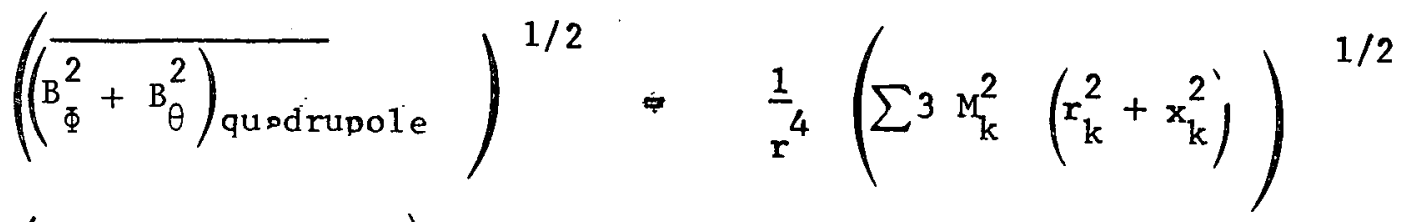

$$
\begin{aligned}
& \left(\overline{\mathrm{B}_{\mathrm{R}}^{2} \text { octupole }}\right)^{1 / 2}=\frac{1}{\mathrm{r}^{5}}\left(\sum{ }^{12 \mathrm{M}_{\mathrm{k}}^{2}}\left(5 \mathrm{x}_{\mathrm{k}}^{4}-2 \mathrm{x}_{\mathrm{k}}^{2} \mathrm{r}_{\mathrm{k}}^{2}+\mathrm{r}_{\mathrm{k}}^{4}\right)^{1 / 2}\right. \\
& \left(\overline{\left.\mathrm{B}_{\Phi}^{2}+\mathrm{B}_{\theta}^{2}\right) \text { octupole }}\right)^{1 / 2}=\frac{1}{\mathrm{r}^{5}}\left(\sum \frac{3}{2} \mathrm{M}_{\mathrm{k}}^{2}\left(5 \mathrm{x}_{\mathrm{k}}^{4}+6 \mathrm{x}_{\mathrm{k}}^{2} \mathrm{r}_{\mathrm{k}}^{2}+5 \mathrm{r}_{\mathrm{k}}^{4}\right)^{1 / 2}\right. \\
& \left(\overline{B_{r, 16-p o l e}^{2}}\right)^{1 / 2}=\frac{i}{r^{6}}\left(\sum \frac{25}{1 ?} M_{k}^{2}\left(175 x_{k}^{6}-165 x_{k}^{4} r_{k}^{2}+45 x^{2} r_{k}^{4}+9 r_{k}^{6}\right)\right)^{1 / 2}
\end{aligned}
$$

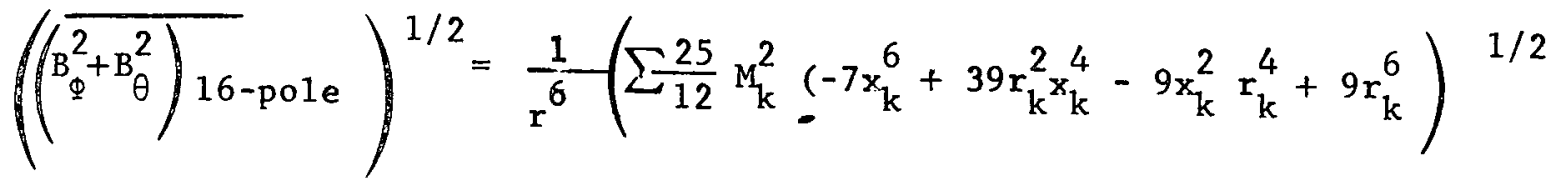

$$
\begin{aligned}
& \left(\overline{B_{R}^{2}, \text { nondipolar }}\right)^{1 / 2}=\left[\sum _ { r ^ { 6 } } \frac { M _ { k } ^ { 2 } } { r ^ { 6 } } \left\{\frac{1}{2}\left(9 x_{k}^{2}+3 r_{k}^{2}\right)\right.\right. \\
& +\frac{48 x_{k}^{3}}{r^{3}}+\frac{1}{r^{4}} \cdot\left(-3 x_{k}^{4}-54 x_{k}^{2} r_{k}^{2}+185 x_{k}^{4}\right) \\
& \left.\left.+0\left(\frac{1}{r^{5}}\right)\right\}\right] 1 / 2 \\
& \left(\overline{\left(B_{\Phi}^{2}+B_{\theta}^{2}\right.} \text { nondipolar }\right)^{1 / 2}=\left[\sum \frac { M _ { k } ^ { 2 } } { r ^ { 6 } } \left\{\frac{3}{r^{2}}\left(x_{k}^{2}+r_{k}^{2}\right)+\frac{12}{3}\left(r_{k}^{2}+x_{k}^{2}\right) x_{k}+\right.\right. \\
& \left.+\frac{1}{r^{4}}\left(-\frac{15}{2} r_{k}^{4}+39 x_{k}^{2} r_{k}^{2}+\frac{65}{2} x_{k}^{4}\right)+o\left(\frac{.1}{5}\right) j\right]^{1 / 2}
\end{aligned}
$$


The bars describe the averaging over an isotropic distribution of dipole orientations. We have chosen the magnetometer boom along the $x$-axis of our coordinate system with $r=|x|$ i.e. the distance from the fixed but arbitrary origin. A sufficient condition for the validity of the expansion leading to equations $1 a$ to $5 b$ is $r>\max \left\{r_{k}\right\}$, where $r_{k}$ is given by $r_{k}=\left(x_{k}^{2}+y_{k}^{2}+z_{k}^{2}\right)^{1 / 2}=\left|x_{k}\right|$. The average of the net nondipolar part cannot be calculated from the single multipole averages alone, since correlations exist between the various multipole contributions.

Several points can be seen from equations la to $5 b$. For the dipole portion of the field, the rms radial component is $\sqrt{2}$ times the rms transverse portion or double the rms - value of $B_{\Phi}$ or $B_{\theta}$. For the. quadrupole part, the ratio can vary statistically between 1 and $\sqrt{2}$ depending on the spatial distribution of sources. Another interesting question is, for what fraction of all possible cases does the $B_{R}$ - component exceed the transverse component $\left(\mathrm{B}_{\Phi}^{2}+\mathrm{B}_{\theta}^{2}\right)^{\mathrm{I}}$ in the far field region i.e. for the dipolar part? Since the dipole moment of the spacecraft field is simply given by $\underline{M}=\sum \underline{M}_{k}$ and the $\underline{M}_{k}^{\prime} s$ are oriented isotropically, the distribution of $M$ must also be isotropic. The fraction of cases with $B_{R}>\left(B_{\Phi}^{2}+B_{\theta}^{2}\right)^{1 / 2}$ is then trivially given as $1-\sqrt{\frac{T}{5}}=0.552786$ independent of the $M_{k}^{\prime} s, N$ and the $\underline{x}_{k}^{\prime} s$.

The above formulas can be used to obtain an estimate of the magnetic field of a spacecraft and particularly of the relative magnitude of its different multipole contributions. The spacecraft is characterized by a distribution of $\mathrm{N}$ randomly oriented magnetic dipoles of strength $\mathrm{M}_{\mathrm{k}}$ and known positions $\underline{x}_{k}$ inside the volume $\mathrm{V}_{\mathrm{sc}}$. In general, this volume 
$V_{\text {sc }}$ can be very complicated, consisting of the spacecraft main body and possible magnetically active appendages. The approximation of random orientations is expected to be a generally reasonable assumption.

The only major exception may be caused by the field of a solar array, which after demagnetization is essentially due to a fairly well known current distribution with known geometry. Thus it may be easily computed using well known principles or it may be negligible due to "backwiring" techniques. Making the latter assumption, which is generally true for spacecraft of recent origin, we can use the formulas la to $5 b$ quite generally.

In addition to using formulas $1 a$ to $5 \mathrm{~b}$ for spacecraft field estimates an important application is to evaluate the instability of a spacecraft field due to changes in the subsystem magnetic fields.

Often it can be assumed that the dipole sources are distributed uniformly in a cube of width $2 a$ or a sphere of diameter $2 a$ in addition to being oriented in a random fashion. Averaging the mean-square magnetic fields in formulas $1 \mathrm{a}$ to $5 \mathrm{~b}$ with respect to position over the volume of the cube with the origin at the center of the cube, we obtain the following rms field components:

$$
\begin{aligned}
& \left.\left(\overline{\left\langle B_{R}^{2}\right. \text { dipole }}\right\rangle\right)^{1 / 2}=\left(\frac{4}{3} \frac{\sum M_{k}^{2}}{r^{6}}\right)^{1 / 2} \\
& \left(\overline{\left.\left\langle\left(B_{\Phi}^{2}+B_{\theta}^{2}\right) \text { dipole }\right\rangle\right)^{1 / 2}=\left(\frac{2}{3} \frac{\sum M_{k}^{2}}{r^{6}}\right)^{1 / 2}}\right.
\end{aligned}
$$




$$
\begin{aligned}
& \left(\left\langle\overline{\mathrm{B}_{\mathrm{R}}^{2}, \text { quadrupole }}\right\rangle\right)^{1 / 2}=\left(\frac{6 \mathbf{a}^{2} \sum \mathrm{M}_{\mathrm{k}}^{2}}{\mathrm{r}^{8}}\right)^{1 / 2} \\
& \left.\left(\overline{\left\langle\left(\mathrm{B}_{\Phi}^{2}+\mathrm{B}_{\theta}^{2}\right)\right. \text { quadrupole }}\right\rangle\right)^{1 / 2}=\left(\frac{4 \mathrm{a}^{2} \sum \mathrm{M}_{\mathrm{k}}^{2}}{\mathrm{r}^{8}}\right)^{1 / 2} \\
& \left(\overline{\left\langle\mathrm{B}_{\mathrm{R}, \text { octupole }}^{2}\right\rangle}\right)^{1 / 2}=\left(\frac{256}{15} \frac{\mathrm{a}^{4} \Sigma \mathrm{M}_{\mathrm{k}}^{2}}{\mathrm{r}^{10}}\right)^{1 / 2} \\
& \left(\left\langle\left(\mathrm{~B}_{\Phi}^{2}+\mathrm{B}_{\theta}^{2},\right)_{\text {octupole }}\right\rangle\right)^{1 / 2}=\left(\frac{222}{15} \cdot \frac{\mathrm{a}^{4} \Sigma \mathrm{M}_{\mathrm{k}}^{2}}{\mathrm{r}^{10}}\right)^{1 / 2} \\
& \left(\overline{\left\langle\mathrm{B}_{\mathrm{R}, 16-\text { pole }}^{2}\right\rangle}\right)^{1 / 2}=\left(\frac{3130}{63} \frac{\mathrm{a}^{6} \sum \mathrm{M}_{\mathrm{k}}^{2}}{\mathrm{r}^{12}}\right)^{1 / 2} \\
& \left(\left(\left(B_{\Phi}^{2}+B_{\theta}^{2}\right) \text { 16-po1e }\right\rangle\right)^{1 / 2}=\left(\frac{2740}{63} \frac{a^{6} \sum M_{k}^{2}}{r^{12}}\right)^{1 / 2} \\
& \left.\left(\overline{\left\langle\mathrm{B}_{\mathrm{R}}^{2},\right. \text { nondipolar }}\right\rangle\right)^{1 / 2}=\left[\frac { \Sigma \mathrm { M } _ { \mathrm { k } } ^ { 2 } } { \mathrm { r } ^ { 6 } } \left\{6\left(\frac{\mathrm{a}}{\mathrm{r}}\right)^{2}+\frac{156}{15}\left(\frac{\mathrm{a}}{\mathrm{r}}\right)^{4}\right.\right. \\
& \left.\left.+0\left(\frac{1}{x^{6}}\right)\right\}\right]^{1 / 2} \\
& \left(\left\langle\left(\mathrm{~B}_{\Phi}^{2}+\mathrm{B}_{\theta}^{2}\right) \text { nondipolar }\right\rangle\right)^{1 / 2}=\left[\frac { \sum M _ { k } ^ { 2 } } { r ^ { 6 } } \left\{4\left(\frac{a}{r}\right)^{2}+\right.\right. \\
& \left.\left.+\frac{202}{15}\left(\frac{a}{r}\right)^{4}+0\left(\frac{1}{r^{6}}\right)\right\}\right]^{1 / 2}
\end{aligned}
$$


A sufficient condition for the convergence of the expansion is $r>\sqrt{3}$ in this case. Averaging over a sphere instead of a cube yields the factors $4 / 3,2 / 3,18 / 5,12 / 5,48 / 7,36 / 7,100 / 9$ and $80 / 9$ inside the square-root on the right side of equations $6 a$ through $9 b$, respectively.

We see, that the rms radial component is always larger than the rms transverse part of the magnetic field. The ratio $\sqrt{\left\langle\mathrm{B}_{\mathrm{R}}^{2}\right\rangle} \sqrt{\left\langle\left(\mathrm{B}_{\Phi}^{2}+\mathrm{B}_{\theta}^{2}\right)\right\rangle}$ decreases as the multipole order increases, however. Close to the spacecraft, i.e., at $r \sim \sqrt{3 a}$, the multipole contributions of low order increase with their order. The transitional

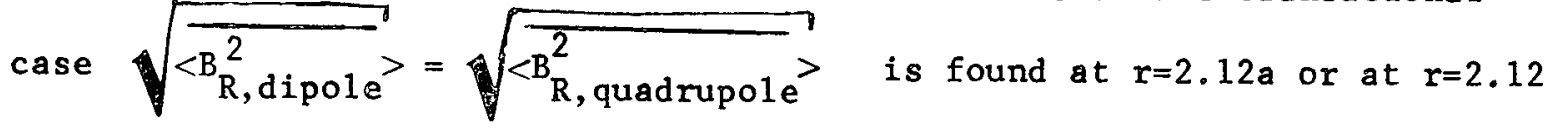
meters for $a=1$ meter, as in section III. A similar result occurs for the transverse field components; the transition occurs at $r=2.45 a$. The equality between the root mean square dipole and octupole fields occurs at $r=1.89 a$ and $r=2.17 a$ for the radial and transverse components, respectively.

It is further interesting, to consider the question beyond which distance do the rms dipole field components dominate the net nondipolar rms-field components? Equations $6 a, 6 b, 10 a$ and $10 \mathrm{~b}$ for the cube show that for the radial component the rms nondipolar field is $~ 56 \%$ of the rms-dipole field at $r=4 a$ and $\approx 37 \%$ at $r=6 a$. For the transverse components the corresponding percentages are $\sim 68 \%$ and $\sim 43 \%$, respectively. At these distances the field is generally strongly dominated by the dipolar portion. Therefore zeros in the spacecraft field components are very improbanle at these distances.

The rms-results for the net nondipolar field contributions with $a=1 \mathrm{~m}$ and $M=1000 \mathrm{ym}^{3}$ are displayed in Fig. 1 and Fig. 2. We not that 


$$
\sqrt{\left\langle\mathrm{B}_{\Phi}^{\overline{2}}\right\rangle}=\sqrt{\left\langle\mathrm{B}_{\theta}^{\overline{2}}\right\rangle} .
$$

The rms field components are proportional to $\sqrt{5 M_{k}^{2}}$. It immediately follows, that the few largest sources determine the magnetic field and not a large number of small sources unless the source distribution falls off very rapidly with source strength. If $M_{k}$ is the same for all $k$, the field components are proportional to $\sqrt{\mathrm{N}}$. Our model also suggests, that a more sensible way to present the results of magnetic cleanliness programs is in terms of (magnetic moment) ${ }^{2}$ per unit mass rather than in terms of the magnetic moment per unit mass. In other words, with the same magnetic moment distribution on the parts-level the net magnetic moment of a spacecraft will be proportional to the square-root of its mass rather than to the mass. 


\section{NUMERICAL ANALYSIS}

For the associated numerical analysis a large number of individual spacecraft field models have been considered with $\mathrm{N}$ dipoles of constant strength $\mathrm{M}=1000 \gamma \mathrm{m}^{3}$ and random orientations and random locations in a cube of volume $8 \mathrm{~m}^{3}$. The nondipolar field portions have been determined by subtracting the pure dipole fields from the total field components, where the dipole field has been obtained from the far field. The nondipolar portions are therefore somewhat inaccurate at large distances.

Figure 1 shows three random samplings for $N=10$ to illust: rate the points made in Section II. It can be seen that the nondipolar parts of $B_{R}$ and $\max \left(B_{\Phi}, B_{\theta}\right)$ and also $\sqrt{B_{R}^{2}+B_{\Phi}^{2}}$ become smaller than the dipolar parts between 2 and $3 \mathrm{~m}$. Figure 2 illustrates the dependence on $\mathrm{N}$ for $\mathrm{N}=1,5,50,100$, which is consistent with a $\sqrt{\mathrm{N}}-1$ aw.

In Fig. 1 and 2 there are two cases with the far-field $\left|B_{R}\right|$ clearly 1arger than $\sqrt{\mathrm{B}_{\Phi}^{2}+\mathrm{B}_{\theta}^{2}}$, one case with the inverse inequality being valid and all other cases showing approximately equality. This is certainly consistent with the statement of $55 \%$ of all cases showing $\left|B_{R}\right|>\sqrt{B_{\Phi}^{2}+B_{\theta}^{2}}$.

The nondipolar fields calculated numerically are generally smaller than the rms nondipolar fields. This is due to the fact that the most probable values are smaller than the average values for the kind of distribution of magnetic field values expected here. This also lends credence to the procedure of using the formulas derived in section II for conservative estimates of the nondipolar parts of a spacecraft field. 
IV. COMPARISON OF SPACECRAFT FIELD MODEL RESULTS WITH MEASURED FIELDS

It is obviously important to consider actual spacecraft magnetic field mappings to ascertain whether an observed spacecraft magnetic field actually possesses any characteristics similar to those described in sections II and III. Unfortunately, only very few cases are available where the spacecraft field has been measured as a function of radial distance along a magnetometer boom. Generally, the field has been measured for various operational modes at the magnetometer position only.

We discuss two examples of measured spacecraft fields which are indicative of the general behavior. Figure 3 shows the results of initial magnetic testing of the IMP-H spacecraft without the kick-motor by Harris (1971, personal communication). The body of the IMP-H spacecraft has the approximate shape of a hexadecagon (16-sided prism) of 1.60 meters length and 1.36 meters diameter. A cube of 1.60 meters edge roughly encloses the whole spacecraft. The results for the cube of 2 meters edge chosen for our model calculations in sections II and III can be scaled down easily to $1.60 \mathrm{~m}$.

Harris (1971, personal communication) reports a variety of tests, the one most representative of the behavior of the spacecraft in space being the powered spacecraft test which includes the perm.magnetic field and the stray magnetic field of the spacecraft. As can be seen from Figure 3 for this case, the radial component $X=B_{R}$ of the field is significantly higher than either of the two transverse components. This is due to the dipole moment lying nearly parallel to the boom. It has components $\mathrm{M}_{\mathrm{x}}=75$ gauss. $\mathrm{cm}^{3}$, $M_{y}=0$ and $M_{z}=10$ gauss. $\mathrm{cm}^{3}$, where the $z$-axis coincides with the spin-axis and the $x$-axis with the boom. The dipolar field contributions are shown 
as dashed 1ines. Harris (1971, persona1 communication) notes that: in

measuring the subsystem fields in many cases the z-component possesses the strongest magnetic field. This is often due to the circuitry being wired in planes perpendicular to the z-exis. This could be taken into account in the previous model by altering the requirement of isotropy of the dipoles. Nevertheless the radial component. still is observed to possess the largest field component.

Inspection of Figure 3 also shows that for the radial component the nondipolar part is certainly much smaller than is given by the ratio calculated from the statistical averages in section II. This result favors the dipolar part because of the particular orientation of the dipole moment, however. A statistica11y more meaningful procedure is to compare the total dipole field, which is $\left(B_{R}^{2} \text {, dipole }+B_{\theta}^{2} \text {, dipole }+B_{\phi}^{2} \text {, dipole }\right)^{1 / 2}$

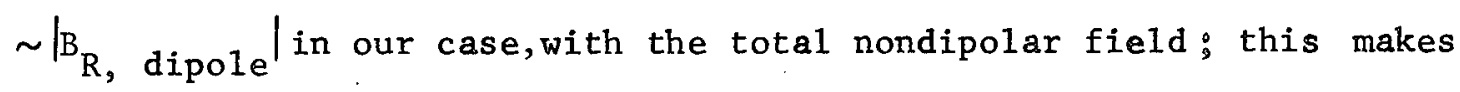
the comparison somewhat independent of the particular dipole orientation. In this more meaningful comparison the nondipolar parts are well within the values from equations $10 a$ and $10 b$, if we use equations $6 a$ and $6 b$ for normaligation.

Because of the relatively large observational errors at distances above about $3 \mathrm{~m}$ this statement is not completely conclusive, however. With respect to the results from IMP-H it should be noted, that due to backwiring and other techniques to achieve "magnetic cleanliness" aboard the spacecraft, the higher order moment field contributions will be relatively enhanced compared with the dipole contributions. The comparison of the model calculations and the observations seems to indicate that equations $7 \mathrm{a}$ through $10 \mathrm{~b}$ could be used to obtain upper bounds on the nondipolar field contributions. The measurements also have shown: that the spacecraft field is very low indeed for IMP-H and that the magnetic clean1iness program was therefore successfu1. 
Another example of spacecraft magnetic fields is provided by Mariner Venus 67. Bastow (1968) reports the following properties concerning the spacecraft magnetic field. The total measured preflight. spacecraft field (stray and perm) was 7.2 gamma with 7.1 gamma pointing in the $z$-direction (nearly radial from the spacecraft center to the magnetometer position). In flight the total spacecraft was estimated to be 10.0 gamma with a $z$-component of 8.7 gamma. The radial component of the field was more than 6 times as large as the transverse field magnitude preflight and nearly twice as large postflight. This again compares well with our model and also indicates the variability of the ratio of the radial and transverse components. 
V. SUMMARY AND CONCLUSIONS

We have presented simple magnetic field models for the dipolar and nondipolar field components of a spacecraft field. The models consist of $N$ dipoles of strength $M_{k}, k=1, \ldots N$, with random orientations and given or random locations. Realizing the large variability of real spacecraft fields, our models suggest the following important properties on the average:

1. The magnetic moment determining the far field is given approximatley by the Pythagorean sum of the individual dipole moments. For equal individual moments the spacecraft magnetic moment is therefore proportional to $\sqrt{\mathrm{N}}$.

2. The radial field component is generally larger than the field transverse to the magnetometer boom. For the far field this is true for $55 \%$ of al1 cases.

3. The dipolar portion of the spacecraft field generally dominates the nondipolar part beyond about $3 a$ from the center where the spacecraft has been represented by a cube of width $2 a$.

4. The spacecraft field is dominated by the few largest sources. 5. These results are consistent with measurements of the field of IMP $\mathrm{H}$ and Mariner 5. 


\section{ACKNOWLEDGMENTS}

We would like to acknowledge helpful discussions with Drs.

Norman F. Ness, K. Behannon and R. L. Lepping concerning this

material. The work of one of us (F.M.N.) has been performed under the NASA-NAS postdoctora1 resident research associateship program. 
- 18 -

FIGURE CAPTIONS

Figure 1: Absolute values of total and nondipolar magnetic field components for three examples picked at random from a statistical ensemble made out of 10 dipoles of strength $\mathrm{M}=1000 \mathrm{\gamma} \cdot \mathrm{cm}^{3}$ isotropically oriented and uniformly distributed in a cube of $2 \mathrm{~m}$ edge as a function of distance along a "boom" from the center of the cube. For comparison the rms nondipolar field components are shown.

Figure 2: Absolute values of total and nondipolar magnetic field components for three examples picked at random from a statistical ensemble made out of 1,5,50,100 dipoles of strength $M=1000 \%, \mathrm{~cm}^{3}$ isotropical1y oriented and uniformly distributed in a cube of $2 \mathrm{~m}$ edge as a function of distance along "boom" from the center of the cube. For comparison the rms nondipolar field components are shown.

Figure 3: Magnetic field distribution along boom of IMP-H during initial testing. The dashed lines show the dipolar parts for each compoent (approximately zero for Y). 
REFERENCES

Bastow, F. G., "The Mariner Venus 67 magnetic field control program", JPL Technical Report 32-1269, Apri1 15, 1968.

Ness, N. F., Magnetometers for space research, Space Sci. Rev., 11, $111-222,1970$.

Ness, N. F., K. W. Behannon, R. P. Lepping and K. H. Schatten, Use of two magnetometers for magnetic field measurements on a spacecraft, J. of Geophys. Res. $\underline{76}, 3564-3573,1971$. 


\section{0}
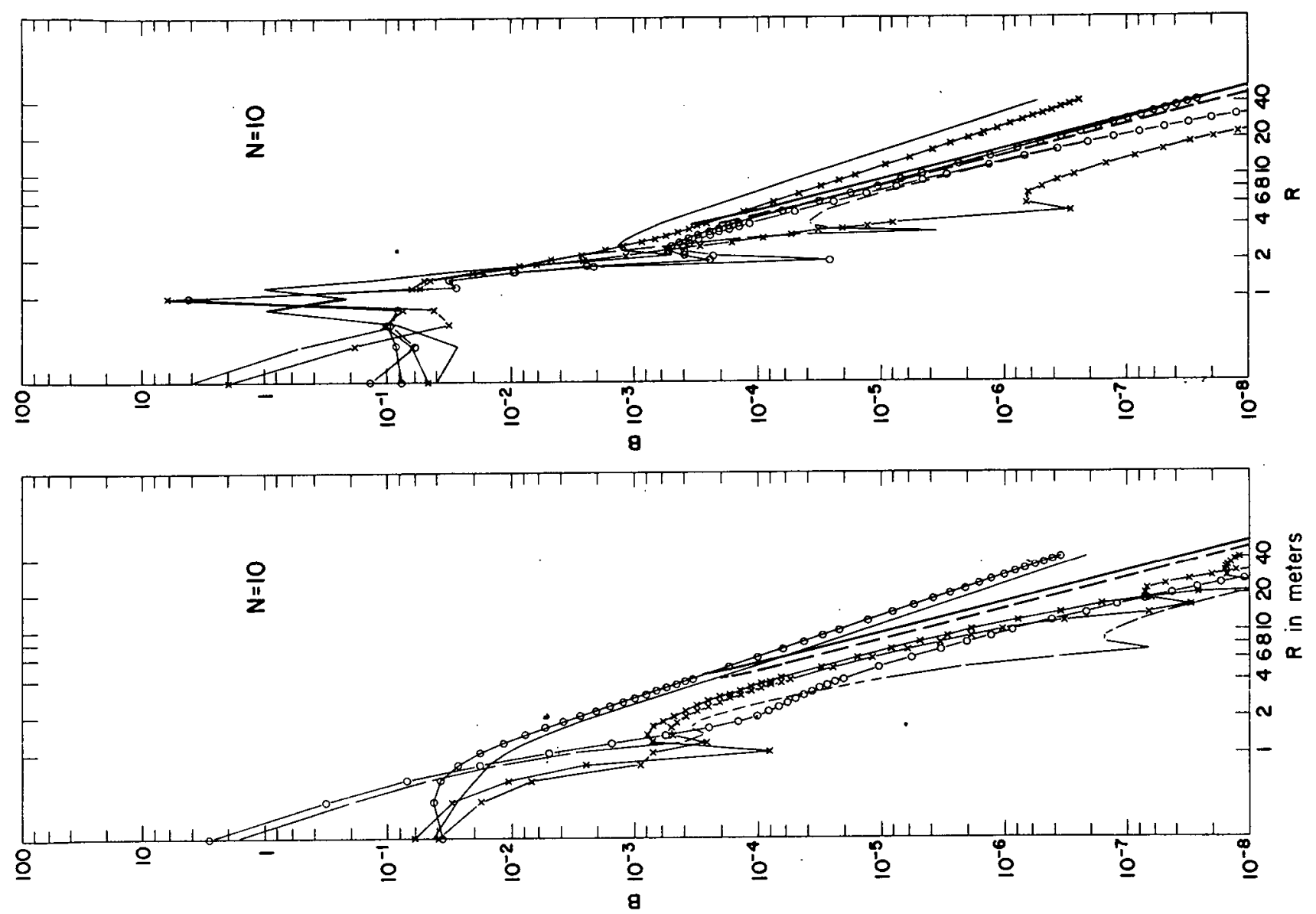

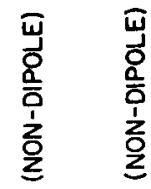
๑ $\left.\right|_{x} ^{x}$ ๓ீ ๓ீ

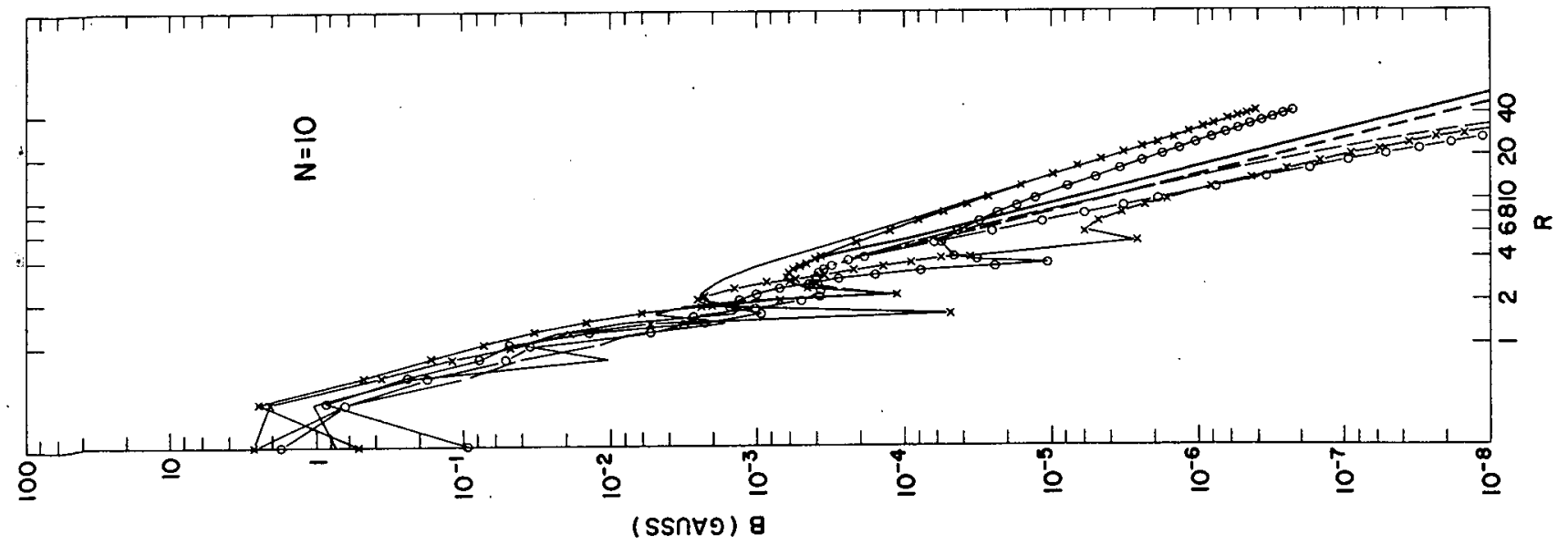

.

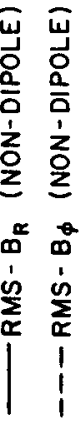


21
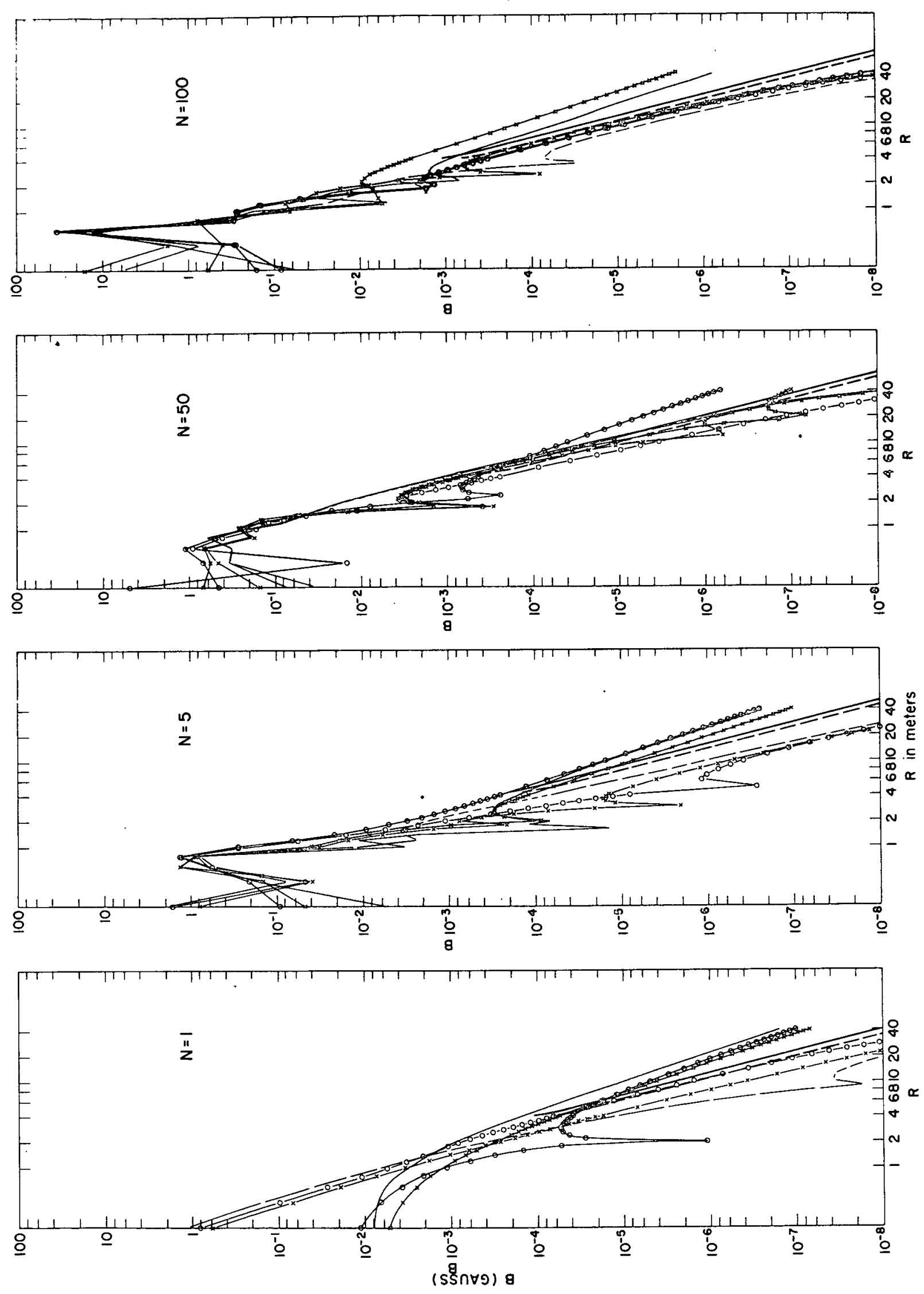
22

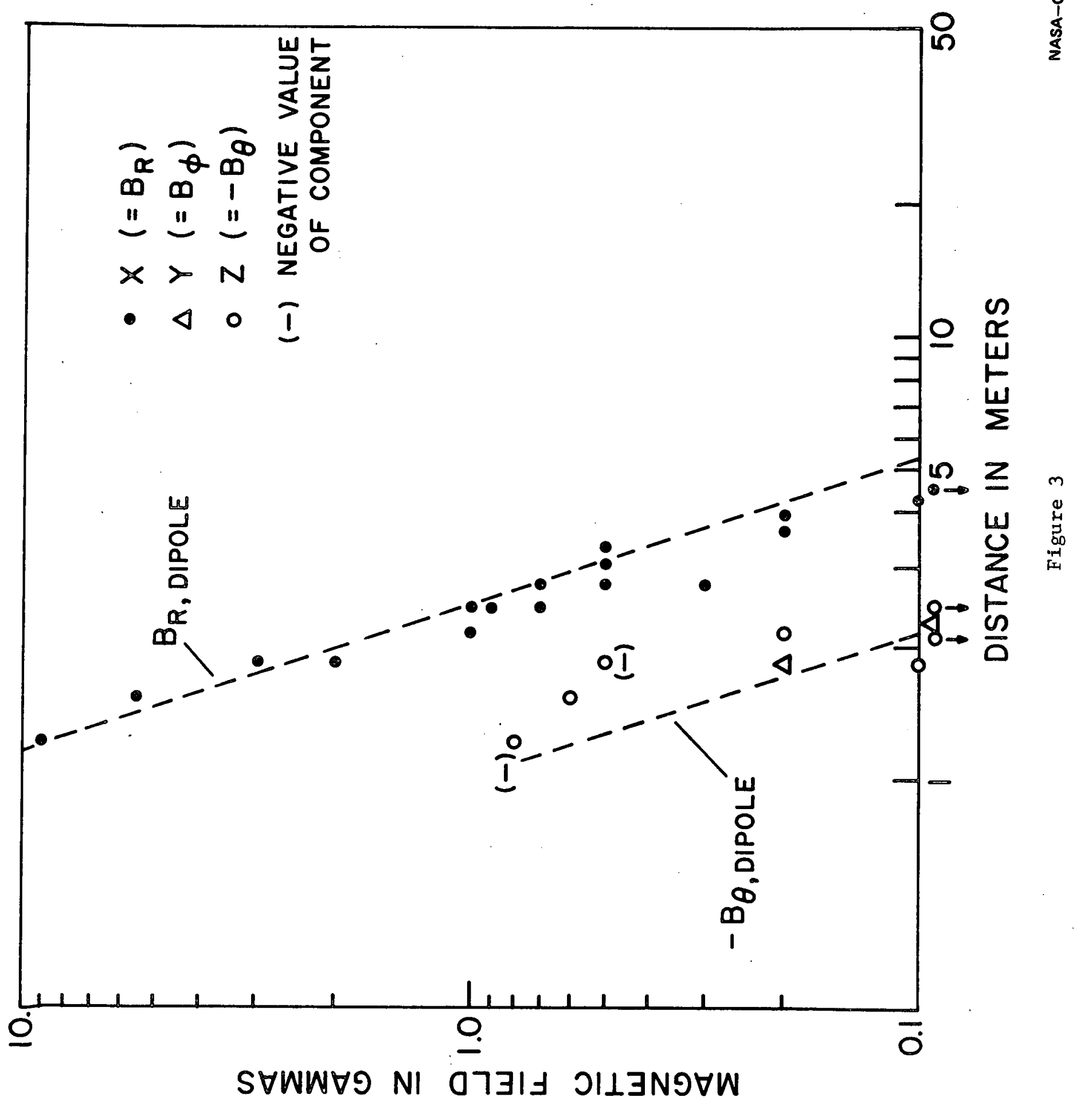

